\title{
Fever and a rash in a 61 year old man
}

\author{
Daniel G Federman, Robert S Kirsner
}

Answers on p243.

Department of
Internal Medicine,
Yale University School
of Medicine, New
Haven and West Haven
VA Medical Center,
West Haven,
Connecticut, USA
D G Federman
Department of
Dermatology and
Cutaneous Surgery,
Department of
Epidemiology and
Public Health,
University of Miami
School of Medicine,
Miami, Florida, USA
R S Kirsner
Correspondence to:
Dr D G Federman, VA
Connecticut Health Care
System (111-GIM), 950
Campbell Ave, West Haven,
CT 06516, USA
Submitted 31 November
1998
Accepted 26 July 1999

A 61 year old white homosexual man complained of profound fatigue, proximal lower extremity weakness, myalgias, and fever of two days' duration. He also complained of a worsening rash over his proximal upper extremities, trunk, and back for the past month. Two months earlier he had been diagnosed with seronegative rheumatoid arthritis when he presented with bilateral hand and wrist pain. He denied cough, shortness of breath, photosensitivity, Raynaud's phenomenon, dry mouth or eyes, recent travel, pets, morning stiffness, penile discharge, or genital ulcers. He tested HIV negative three months before admission. His past medical history was significant for hypertension, gastro-oesophageal reflux disease, and obstructive sleep apnoea. He had been taking prednisone $15 \mathrm{mg}$ and cimetidine $400 \mathrm{mg}$ daily.

On examination, the patient looked acutely ill and uncomfortable. He was febrile at $102.4^{\circ} \mathrm{F}$, his blood pressure was $148 / 80 \mathrm{~mm}$ $\mathrm{Hg}$, and his pulse was 90 beats/min. He had confluent erythematous and purpuric plaques on his back, anterior trunk, and proximal upper extremities (see fig 1); some were in a polycyclic arrangement but without scale. No muscle tenderness or weakness was detected. There was no appreciable arthritis or synovial thickening. He had a II/VI systolic murmur throughout his precordium without radiation. The remainder of his physical examination was without abnormalities.

The initial evaluation is listed in box 1. A skin biopsy showed superficial and deep perivascular infiltrates with extensive interface damage. Immunofluorescence revealed strong

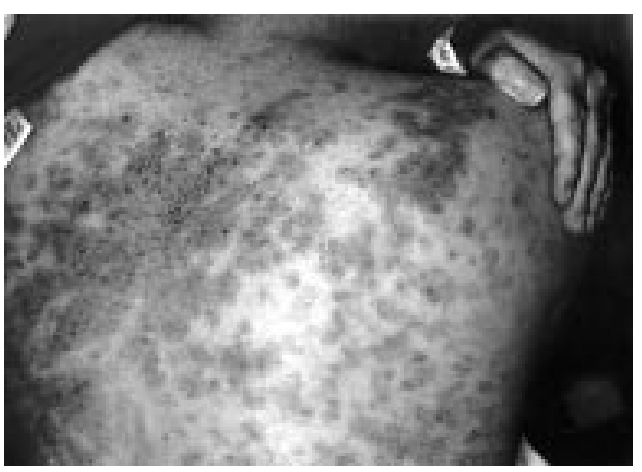

Figure 1 Purpuric plaques. granular C3 and trace granular IgG and $\operatorname{IgM}$ at the dermal-epidermal junction.

\section{Questions}

(1) What diagnoses would be consistent with the results of the skin biopsy?

(2) What are the cutaneous lesions of lupus erythematosus?

(3) Does he meet criteria for the diagnosis of systemic lupus erythematosus (SLE)?

(4) How common is SLE in patients of this age, race, and gender?

(5) As the patient was at risk for HIV infection, what dermatological manifestations of HIV should be considered?

\section{Box 1: Initial evaluation}

White blood count $=2.6 \times 10^{9} / 1$

Haemoglobin $=145 \mathrm{~g} / 1$

Platelet count $=143 \times 10^{9} / 1$

Electrolytes, blood urea nitrogen, creatinine, urinalysis, calcium, albumin $=$ normal

Alkaline phosphatase, bilirubin normal Aspartate aminotransferase $=60 \mathrm{U} / \mathrm{L}$ Alanine aminotransferase $=56 \mathrm{U} / \mathrm{L}$

Aldolase, creatine phosphokinase normal

Erythrocyte sedimentation rate $=50$ $\mathrm{mm} /$ hour

Antinuclear antibody positive at 1:160

IgM anticardiolipin antibody positive

Rheumatoid factor positive at 1:1280

Venereal Disease Research Laboratory non-reactive

Anti-Ro, anti-La, antiribonuclear protein, anti-Sm, antidouble stranded DNA, anticentromere antibodies negative

C3, C4, CH50 concentrations normal

Quantitative serum HIV RNA by polymerase chain reaction negative

Blood cultures negative

Electromyography with nerve conduction studies normal 


\section{Painful knee: a dilemma}

A A Syed

Answers on p244.

A 55 year old postmenopausal female, previously in good health, had an abrupt onset of right knee pain. This was managed by her general practitioner for three months, with analgesics and non-steroidal anti-inflammatory drugs (NSAIDs). She had no recollection of any trauma. As the symptoms failed to resolve, she was referred to the orthopaedic service. On history, the knee pain was deep seated, sharp, and intermittent. It was made worse on exertion and relieved by rest. There was no associated musculoskeletal or neurological abnormality. On physical examination, she looked well. The knee joint appeared normal without any restriction of motion. All movements of her lumbar spine were normal. Examination of the hip joint was negative except for painful restriction of internal and external rotation. Blood tests including full blood count, erythrocyte sedimentation rate, and bone profile were normal. Specific tests for rheumatoid factor, antinuclear antibodies, brucella antigen, HLA B-27, and thyroid function tests were all negative. Radiographic examination of the lumbar spine, knee, and the hip (fig 1) revealed no abnormality. An isotope bone scan of the pelvis is shown (fig 2). Arthrocentesis of the hip joint yielded few millilitres of clear yellow fluid with no bacteria seen on acid-fast, fungal, or Gram stains. Culture for aerobic, anaerobic, acid-fast, and fungal organisms was also negative. A bone biopsy under general anaesthesia gave unremarkable results. Finally,

Cappagh Orthopaedic Hospital, Dublin 11 Republic of Ireland

Correspondence to: Mr A A Syed, Department of Orthopaedics, Bradford Royal Infirmary, Bradford BD9 6RJ, UK

Submitted 15 January 1999 Accepted 5 July 1999

Figure 1 Radiograph of the pelvis at three months since onset of symptoms. a magnetic resonance imaging (MRI) scan was conducted (fig 3).

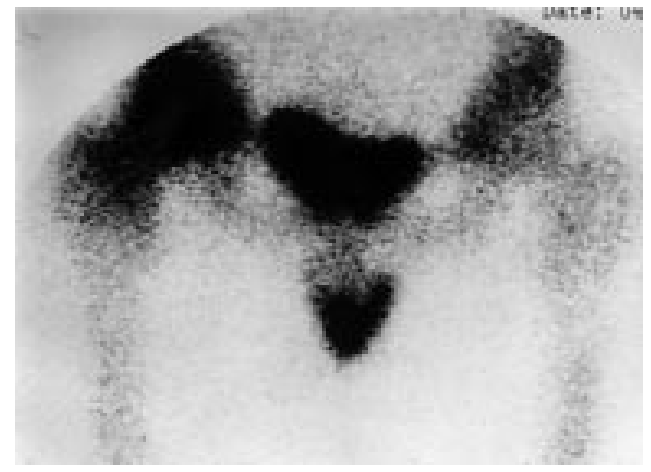

Figure 2 Radionuclide bone scan of the pelvis.
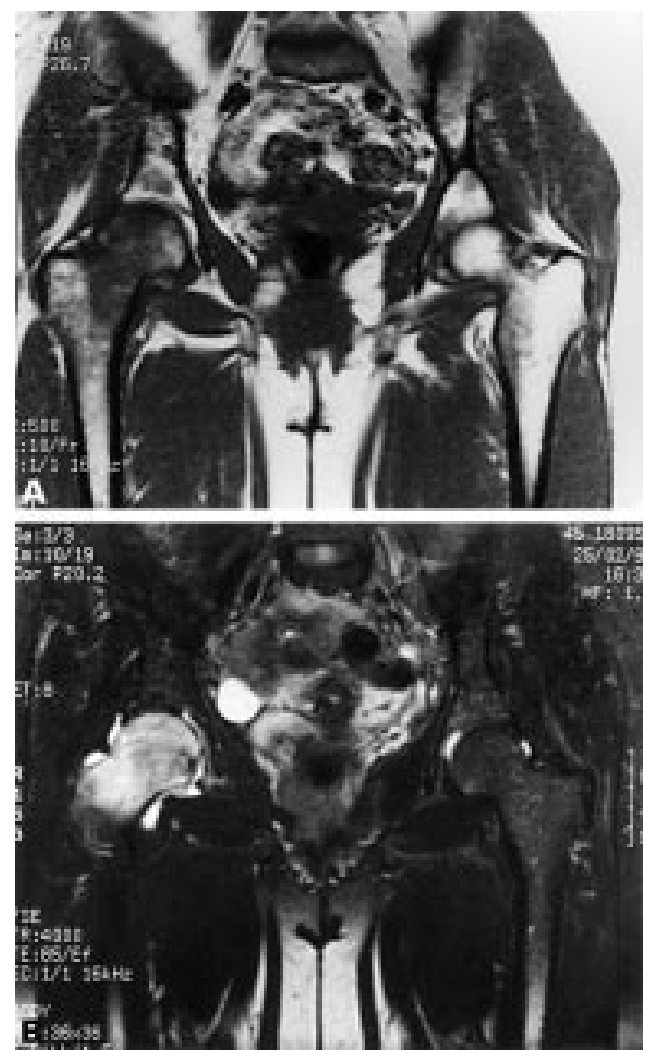

Figure 3 (A) Coronal T1 weighted MRI image and (B) coronal T2 weighted MRI image.

\section{Questions}

(1) What are the findings on the bone scan and the MRI scans? 


\title{
Back pain and dyspnoea in a middle aged diabetic male
}

\author{
J Kelly, G Thorning, A Ozzard, K Kelleher
}

Answers on $p 245$.

A 59 year old insulin dependent diabetic male presented with an eight week history of lower thoracic back pain and a two to three week history of progressive exertional dyspnoea, limiting his exercise tolerance to around 100 yards, associated with mild ankle oedema. He did not complain of cough or chest pain. He had been discharged from another hospital four weeks earlier after a prolonged admission with pneumonia and renal failure.

There was no other past medical history of note. In particular, there was no history of cardiac disease. He was taking insulin and enalapril.

On examination, he was comfortable at rest though appeared chronically unwell. Temperature was $38.5^{\circ} \mathrm{C}$. there were no cutaneous stigmata of endocarditis. Cardiovascular examination revealed a resting tachycardia of 120 beats/min, a gallop rhythm, systolic and diastolic murmur over the pulmonary area, and mild bilateral ankle oedema. Chest auscultation revealed bilateral basal rales. Abdominal and neurological examinations were unremarkable. There was tenderness over the lower thoracic spine.

Blood results are shown in box 1 .

Box 1: Blood results (normal range in
parentheses)
Sodium, potassium normal
Urea $=11.4 \mathrm{mmol} / 1(2.5-6.5)$
Creatinine $=158 \mu \mathrm{mol} / 1(40-110)$
Glucose $=9.1 \mathrm{mmol} / 1(3-6.7)$
Albumin $=30 \mathrm{~g} / 1(30-52)$
Globulins $=54 \mathrm{~g} / 1(18-35)$
Calcium, other liver function tests and
thyroid function tests normal
Haemoglobin $=91 \mathrm{~g} / 1(130-180)$
Mean corpuscular volume $=83 \mathrm{fl}(80-100)$
White blood cells $=19.410^{9} / 1(4-11)$
Neutrophils $=16.210^{9} / 1(2-7.5)$
Clotting screen normal
Erythrocyte sedimentation rate $=144$
mm/hour
Arterial blood gases $($ room air):
pH = 7.49 $(7.35-7.45)$
Carbon dioxide pressure $=3.3 \mathrm{kPa}$
$\quad(4.7-6.0)$
Oxygen pressure $=8.9 \mathrm{kPa}(>10.6)$
Oxygen saturation $=94 \%$
Bicarbonate $=19 \mathrm{mmol} / 1(24-30)$

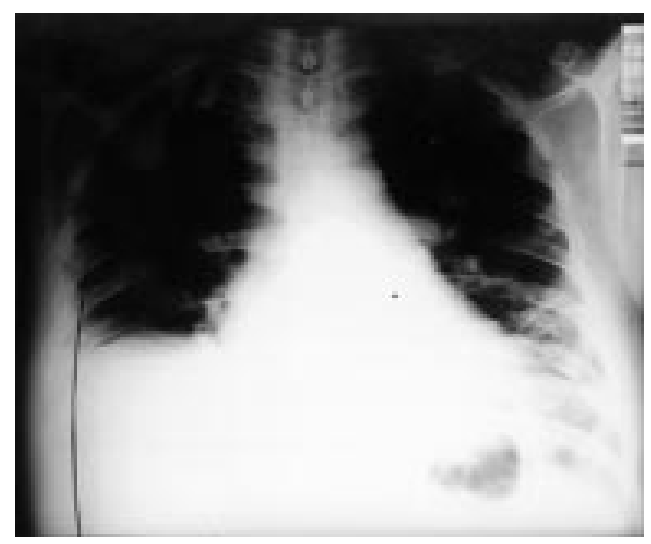

Figure 1 Chest radiograph.

Chest radiography showed cardiomegaly with patchy shadowing at both bases (fig 1). The electrocardiogram showed sinus tachycardia with right bundle branch block.

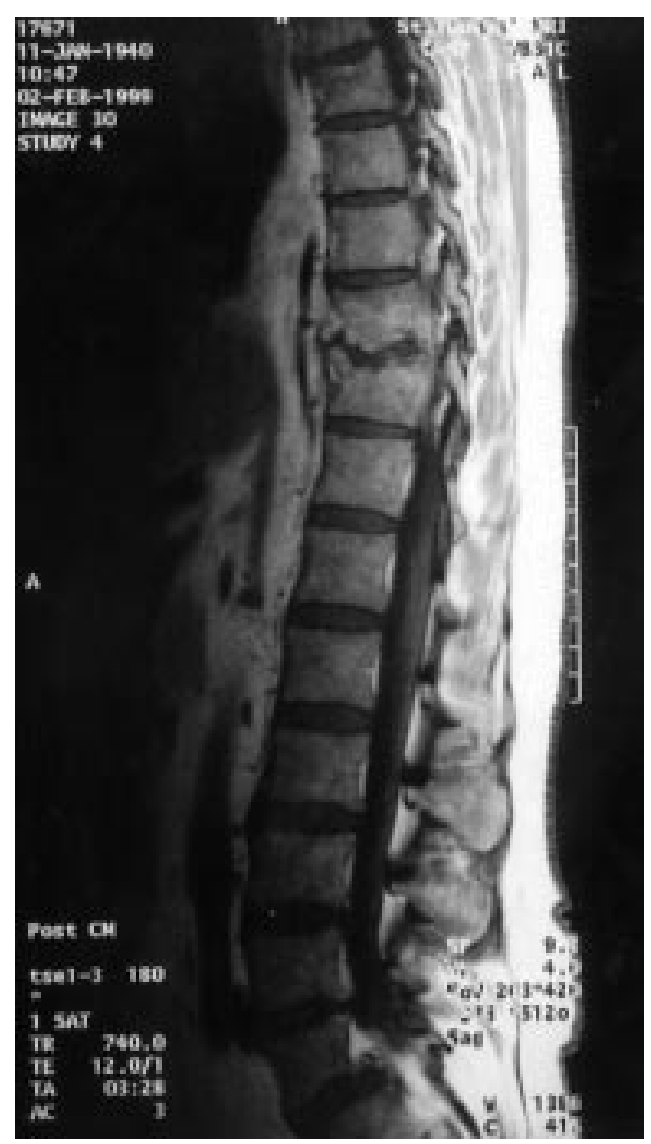

Figure 2 Gadolinium enhanced magnetic resonance imaging scan $T 1$ and $T 2$ weighted sagittal acquisitions of lower thoracic spine.
Queen Mary's Hospital, Sidcup, Kent, UK

J Kelly

G Thorning

A Ozzard

K Kelleher

Correspondence to: Dr James Kelly, Northbrook, Fairmile Lane, Cobham,
Surrey KT11 2DQ, UK

Submitted 8 July 1999 Accepted 13 August 1999
Bicarbonate $=19 \mathrm{mmol} / \mathrm{l}(24-30)$ 


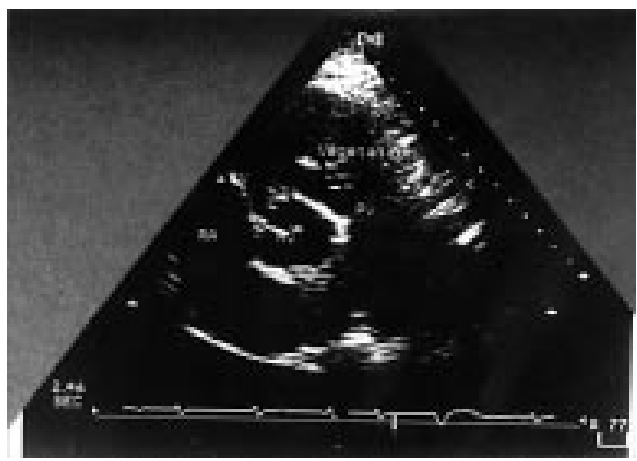

Figure 3 Appearance of pulmonary valve on a transthoracic echocardiogram.

Given the history of back pain with hyperglobulinaemia and very high erythrocyte sedimentation rate, multiple myeloma was considered likely complicated either by a community acquired bibasal pneumonia or multiple pul- monary emboli. Two sets of blood cultures were taken and the patient started on subcutaneous low molecular weight heparin and antibiotics while awaiting urgent echocardiography and VQ scanning.

The next day, Staphylococcus aureus was cultured from all four blood culture bottles. The appearance of a magnetic resonance imaging scan of the thoracic spine and transthoracic echocardiogram are shown in figs 2 and 3.

\section{Questions}

(1) What is the unifying diagnosis?

(2) How does right sided endocarditis usually present?

(3) What risk factors are recognised for right sided endocarditis in non-intravenous drug users?

(4) What is the likelihood of endocarditis in cases of $S$ aureus bacteraemia?

(5) What is the relationship between back pain and endocarditis?

\section{A man with abdominal pain}

\author{
K H Siddiqui, J G H Hubbard
}

Answers on p248.

Sunderland Royal Hospital, Kayll Road, Sunderland, UK K H Siddiqui J G H Hubbard

Correspondence to: Mr JGH Hubbard, North Tees General Hospital, Hardwick, Stockton-on-Tees TS19 8PE, UK

Submitted 10 June 1998 Accepted 23 June 1999
A 44 year old man previously in good health presented to the accident and emergency department with a 3-day history of gradually worsening abdominal pain. The pain started as a dull ache in the periumbilical region and later became diffuse and more severe. $\mathrm{He}$ developed diarrhoea 24 hours after the pain started, opening his bowels up to 10 times a day. This was initially brown watery motion but later became mixed with dark blood. There was associated vomiting and anuria.

On examination he was mildly jaundiced and dehydrated. His temperature was $39^{\circ} \mathrm{C}$. His pulse was irregular at a rate of 120 beats/min, blood pressure 95/40 $\mathrm{mm} \mathrm{Hg}$. His abdomen was distended and diffusely tender, rectal examination was unremarkable. Initial investigations showed a haemoglobin of $17.9 \mathrm{~g} / \mathrm{dl}$, white cell count $24 \times 10^{9} / 1$, urea 22 $\mu \mathrm{mol} / 1$, creatinine $200 \mu \mathrm{mol} / 1$, bilirubin 29 $\mu \mathrm{mol} / \mathrm{l}$ and alkaline phosphatase $308 \mathrm{IU} / 1$; urinalysis, electrolytes and amylase were otherwise normal. Blood gases showed a mild metabolic acidosis and electrocardiogram confirmed the presence of atrial fibrillation. Chest $x$-ray was normal; abdominal $x$-ray is shown in fig 1.

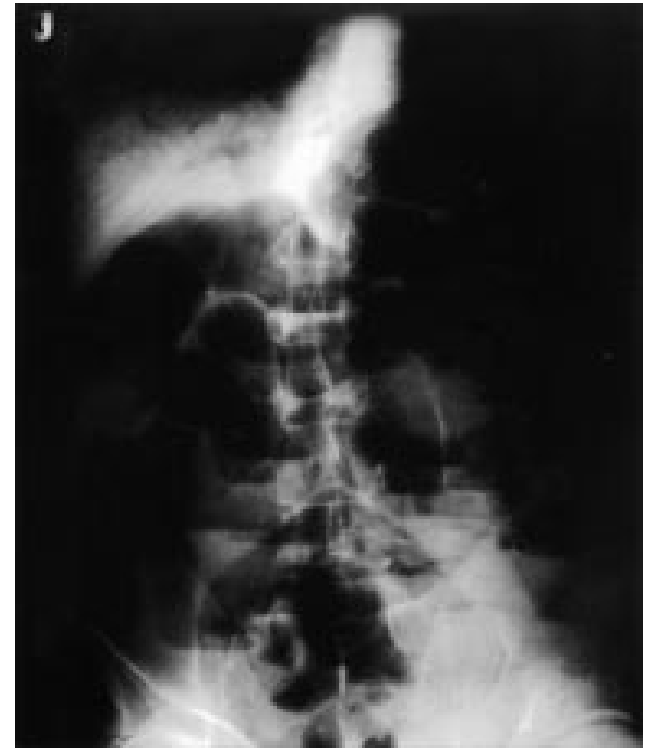

Figure 1 Abdominal x-ray.

\section{Questions}

(1) What is the unusual feature on the plain abdominal $x$-ray?

(2) What are the causes of this feature?

(3) Is laparotomy indicated? 


\title{
A shot in the dark
}

Answers on $p 249$.

\author{
N R Bees, J V Cook
}
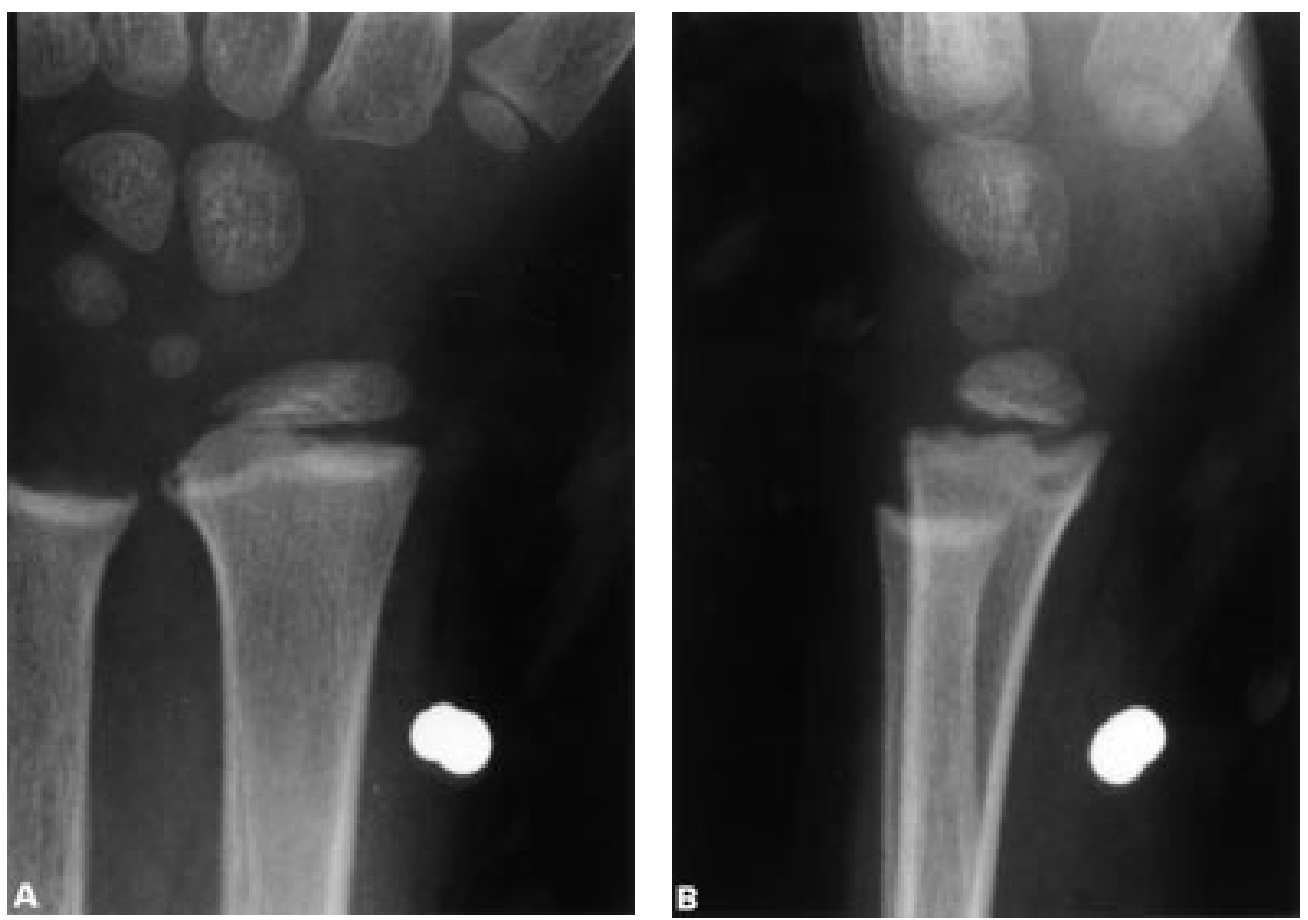

Department of

Radiology, Queen

Mary's Hospital for

Children, Wrythe

Figure 1 Dorsopalmar and lateral radiographs of the left wrist.

Maulana Azad Medical College and Lok Nayak Hospital, New Delhi, India: Department of Pathology

Z N Singh

S Rani

S Jain

Department of

Medicine

S K Jain

Correspondence to: Dr Zeba Niazi Singh, D-2 2276 Vasant Kunj, New Delhi

Submitted 22 March 1999 Accepted 23 June 1999

A 9 year old boy of short stature presented to accident and emergency with trauma to his left wrist following an accident with an air gun (fig 1).

\section{Questions}

(1) What abnormalities are demonstrated on the plain radiographs of the wrist (fig 1)?
(2) What biochemical tests would aid the diagnosis?

(3) What additional radiological investigation should be performed and what abnormalities might be expected?

\section{Pyrexia and pancytopenia with unusual host immune response}

\section{Zeba Niazi Singh, Shyama Jain, S K Jain, Sudha Rani}

A 50 year old man presented to the emergency department in a febrile drowsy state. His relatives gave a history of continuous moderate grade fever up to $38^{\circ} \mathrm{C}$ for the last 2 months. Occasional chills, cough with scanty mucoid expectoration, anorexia and weight loss were also present. He was a farmer by profession, non-smoker, non-alcoholic. There was no history of drug abuse, intravenous injections, tuberculosis or diabetes mellitus. Sexual history could not be elicited. Physical examination revealed a conscious but drowsy patient, with marked pallor and moderate hepatosplenomegaly. Cyanosis, petechiae and peripheral lymphadenopathy were absent. Respiratory and cardiovascular system were normal on examination. No focal deficit was elicited in the central nervous system. Blood examination revealed a haemoglobin of $6.5 \mathrm{~g} / \mathrm{dl}$, total leucocyte count of $1.4 \times 10^{9} / 1$ and platelet count of $40 \times 10^{9} / 1$. The red cells were normocytic normochromic, no abnormal cells were identified on the peripheral smear. Biochemical profile was within normal limits. Bedside chest $x$-ray 


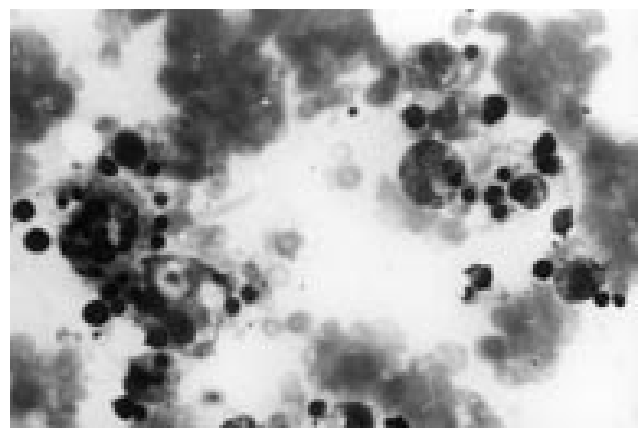

Figure 1 Photomicrograph of the bone marrow aspirate (Giemsa orig $\times 40)$.

did not show any lesion. A bone marrow aspiration was performed (fig 1 and $2 \mathrm{~A}$ ).

The patient deteriorated during the 12 hours of hospital stay. Despite supportive therapy, he developed petechial skin rashes all over the body and started bleeding from the site of arterial puncture. He also had upper and lower gastrointestinal bleed, became hypotensive and expired. A postmortem splenic aspiration was also performed (fig $2 \mathrm{~B}$ ).

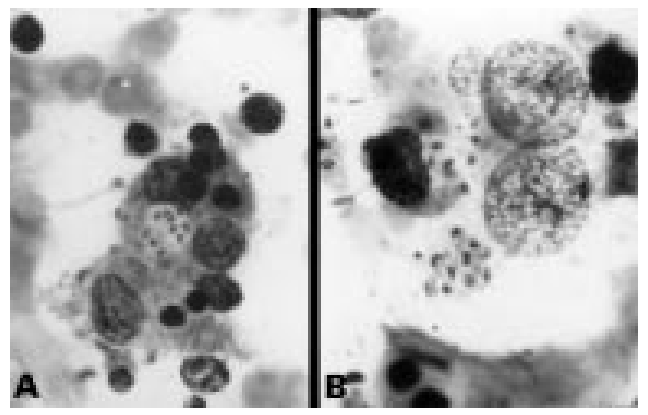

Figure 2 Photomicrograph of $(A)$ bone marrow aspirate smear; (B) splenic aspirate (Giemsa orig $\times 100)$.

\section{Questions}

(1) What cytomorphological phenomenon is shown in fig 1 ?

(2) List the conditions with which this cytomorphological phenomenon may be associated.

(3) What is the diagnosis in this case?

\title{
Abdominal colic after vigorous exercise in a middle aged man
}

\author{
A G Speers, S K Das
}

Answers on $p 252$

\section{Department of Vascular Surgery, Frimley Park Hospital Camberley, UK A G Speers S K Das \\ Correspondence to: Mr S K Das, Hillingdon Hospital, Pield Heath Road, Uxbridge, Middlesex B8 3NN, UK \\ Submitted 9 March 1999 Accepted 13 August 1999}

A 39 year old Army recruit and a long distance runner was referred to the vascular clinic with a two year history of abdominal colic after vigorous exercise (typically running for greater than 40 minutes). It was described as cramp like with a heavy feeling in his guts. The pain resolved with rest but was associated with increased flatulence, nausea, and fatigue. The pain was not demonstrated when he ate a heavy meal; there was no history of diarrhoea or weight loss.

Three years before the patient had undergone coronary artery bypass grafting for angina, New York Heart Association class II. There was a positive history of familial hypercholesterolaemia and ischaemic heart disease. His serum cholesterol was initially $11.1 \mathrm{mg} / \mathrm{l}$ at presentation but was brought down by medical

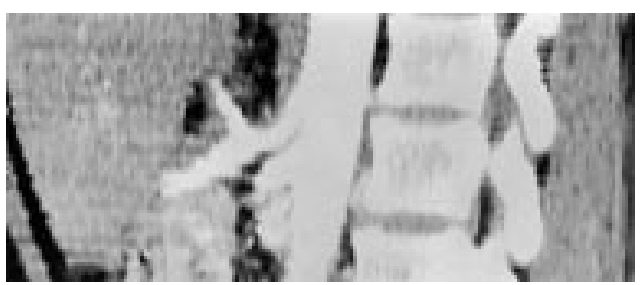

Figure 1 Computed tomogram of the abdominal aorta and coeliac axis demonstrating a tight stenosis at the origin of the coeliac artery with post-stenotic dilatation. The superior mesenteric artery is seen to be of normal calibre.

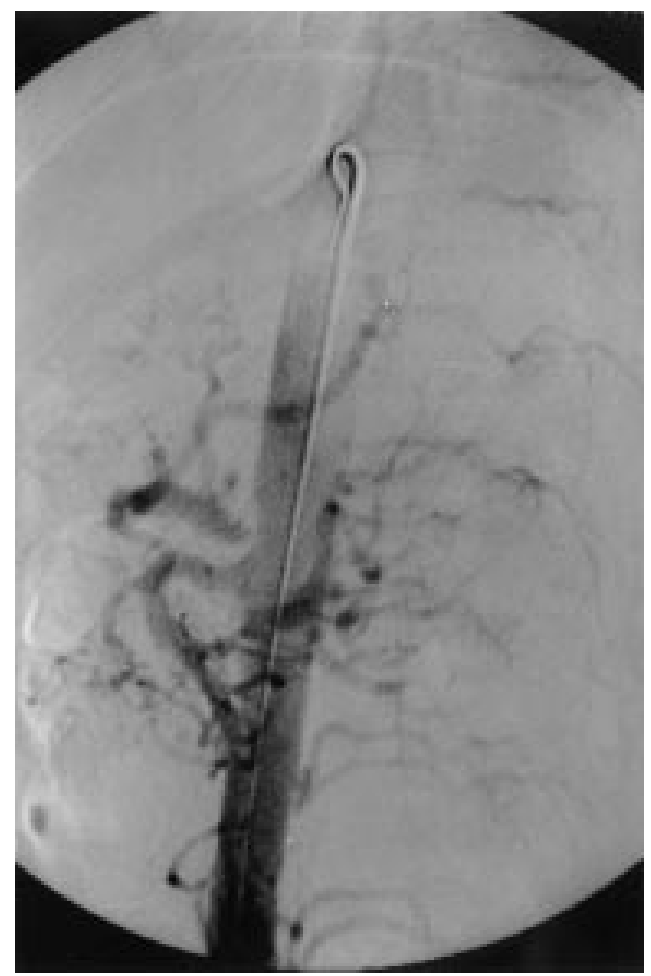

Figure 2 Aortogram showing aortic atheroma with a tight stenosis at the origin of the coeliac artery and post-stenotic dilatation. The superior mesenteric artery is of normal calibre. 
and dietary measures. He was a non-smoker. On examination the patient looked extremely healthy; blood examination revealed a mildly raised cholesterol concentration $(6.2 \mathrm{mg} / \mathrm{l})$. An ultrasound examination for urinary and biliary tract was normal. A barium meal study did not reveal any abnormality in the upper gastrointestinal tract. A duplex scan of the arterial tree with particular emphasis on the coeliac and mesenteric vessels did not reveal any abnormality. This was followed by contrast computed tomography (fig 1) and mesenteric angiogram (fig 2).

\section{Questions}

(1) What is the diagnosis?

(2) What are the differential diagnoses in this patient?

(3) How would you establish the diagnosis?

(4) What do the investigations illustrate?

(5) What are the treatment options available?

\section{SELF ASSESSMENT ANSWERS}

\section{Fever and rash in a 61 year old man}

Q1: What diagnoses would be consistent with the results of the skin biopsy?

The biopsy findings in this patient are not specific for one disease entity, but may be found in lupus erythematosus, dermatomyositis, as well as some cutaneous drug reactions, and in acute graft versus host disease. Given his constellation of features, as well as his normal neuromuscular examination, muscle enzymes and electromyography results, and the uncommon frequency of cutaneous reactions to prednisone or cimetidine, a clinical diagnosis of lupus erythematosus was made.

\section{Q2: What are the cutaneous lesions of lupus erythematous?}

Lupus erythematosus may be limited to the skin or may exist as part of a multisystem disease, systemic lupus erythematosus (SLE). Chronic cutaneous lupus erythematosus lesions include discoid lesions, lupus panniculitis, or hypertrophic (verrucous) lupus erythematosus. The two most common types of subacute cutaneous lupus erythematosus are the psoriasiform (papulosquamous) and annular variants. Approximately $70 \%$ of these individuals have the anti-Ro (SS-A) antibody. ${ }^{1}$ Cutaneous lesions seen in acute lupus include malar erythema, photosensitivity, papular palmar erythema, periungual erythema, Raynaud's phenomenon, diffuse hair loss (telogen effluvium), urticarial vasculitis, leukocytoclastic vasculitis, and bullous lesions. The frequency of systemic involvement is related to the subtype of cutaneous lupus erythematosus and is more common in acute lupus erythematosus (100\% SLE), than in subacute lupus erythematosus $(50 \%)$, and chronic lupus erythematosus $(5 \%-10 \%)$

\section{(3) Does he meet criteria for the} diagnosis of SLE?

According to the 1997 criteria by the American College of Rheumatology, ${ }^{2}$ a diagnosis of SLE can be made when four of the 11 criteria are fulfilled (box 2). Our patient initially met four of the criteria.

Q4: How common is SLE in patients of this age, race, and gender?
SLE is more common in females, who are afflicted almost four times as often as men. Black women are three times more likely to develop SLE than white women, while black men develop SLE 1.5 times more often than white men. ${ }^{3}$ Although SLE is much more common in younger individuals, approximately $10 \%$ of patients are diagnosed when older than $60 .{ }^{4}$

\section{Box 2: 1997 American College of Rheumatology criteria for the classification of SLE}

(1) Malar rash: fixed erythem, flat or raised, over the malar eminences.

(2) Discoid rash: erythematous raised patches with adherent keratotic scaling and follicular plugging.

(3) Photosensitivity.

(4) Oral ulcers: oral or nasopharyngeal.

(5) Arthritis: non-erosive arthritis involving two or more peripheral joints.

(6) Serositis: pleuritis or pericarditis.

(7) Renal disorder: proteinuria $>0.5 \mathrm{~g} /$ day or greater than $3+$, or cellular casts.

(8) Neurological disorder: seizures or psychosis without other cause.

(9) Haematological disorder: haemolytic anaemia or leukopenia $\left(<4.0 \times 10^{9} / 1\right)$ or lymphopenia $\left(<1500 \times 10^{6} / 1\right)$ or thrombocytopenia $\left(<100 \times 10^{9} / 1\right)$.

(10) Immunologic disorder: anti-ds DNA or anti-Sm or a positive finding of anitphospholipid antibodies based on (I) an abnormal serum level of IgG or IgM anticardiolipin antibodies, (ii) a positive test result for lupus anticoagulant, or (iii) a false positive serologic test for syphilis.

(11) Antinuclear antibodies: an abnormal titre of antinuclear antibodies by immunofluorscence or an equivalent assay at any point in time in the absence of drugs known to induce antinuclear antibodies. 
Q5: As the patient was at risk for HIV infection, what dermatological manifestations of HIV should be considered?

As the patient had been recently tested for HIV and was negative, it would be unlikely that his findings were caused by advanced HIV disease. Patients with acute HIV infection might experience an acute retroviral syndrome, which consists of fever, sweats, arthralgias, myalgias, lymphadenopathy, headache, and a maculopapular eruption on the trunk, face, and upper extremities. An enanthema is also frequently present. The clinical and histological appearance of these lesions are not easily confused with the patient's lesions.

\section{Discussion}

There are a variety of cutaneous manifestations of lupus erythematosus. The type of lesion may help predict a patient's course. Our patient's cutaneous lesions were most consistent with subacute cutaneous lupus erythematosus despite the negative anti-Ro antibodies. Originally described in a cohort of 27 patients by Sontheimer and Gilliam in $1979,{ }^{5}$ subacute cutaneous lupus erythematosus was found to be associated with alopecia (59\%), photosensitivity (52\%), mucus membrane ulcers (37\%), and facial telangiectasias (30\%). Livedo reticularis, periungual telangiectasias, discoid lesions, vasculitis, and sclerosis were each found in less than $25 \%$ of their patients. Arthritis or arthralgia $(74 \%)$, fever or malaise $(37 \%)$, and myalgia $(22 \%)$ were the most common systemic manifestations of subacute cutaneous lupus erythematosus. Although central nervous system and renal disease were seen in 19\% and $11 \%$ respectively, the frequency and severity of involvement is less than in acute lupus. Sixty three per cent of their patients had an antinuclear antibody titre of greater than or equal to $1: 160,59 \%$ had an erythrocyte sedimentation rate of greater than $30 \mathrm{~mm} /$ hour, $20 \%$ had a positive rheumatoid factor, while only $37 \%$ were found to have a positive antidouble stranded DNA antibody test. This contrasts to acute SLE, where the antinuclear antibody is positive in greater than $95 \%$ of cases, and the antidouble stranded DNA antibody is positive in $60 \%$ to $80 \%$ of cases. ${ }^{6}$

Although approximately $70 \%$ of subacute cutaneous lupus erythematosus patients will be found to possess the anti-Ro antibody, our patient was found to be anti-Ro negative. Our patient could be part of a subset of patients with subacute cutaneous lupus erythematosus that do not posses the anti-Ro antibody. Alternatively, titres may fluctuate and perhaps with time his anti-Ro antibody will become detectable.

Our patient was initially treated with $60 \mathrm{mg}$ of prednisone daily and $200 \mathrm{mg}$ of hydroxychloroquine twice daily. Within 72 hours, his systemic manifestations were greatly improved. Over the ensuing three months, his skin lesions completely resolved. Interestingly, as the prednisone dose was slowly tapered, he developed oral ulcers approximately three weeks after his discharge from the hospital.
Final diagnosis

Subacute cutaneous lupus erythematosus.

1 Callen JP, Tuffanelli DL, Provost TT. Collagen-vascular disease: an update. 7 Am Acad Dermatol 1993;28:477-84.

2 disease: an update. 7 Am Acad Dermatol 1993;28:477-84. tolgy revised criteria for the classification of systemic lupus erythematosus. Arthritis Rheum 1997;40:1725.

3 Kaslow RA, Masi AT. Age, sex, and race effects on mortality from systemic lupus erythematosus in the United States. Arthritis Rheum 1978;21:473.

4 Von Feldt JM. Systemic lupus erythematosus. Recognizing its various presentations. Postgrad Med 1995;97:79-94.

5 Sontheimer RD, Thomas JR, Gilliam JN. Subacute cutaneous lupus erythematosus. A cutaneous marker for a distinct lupus erythematosus subset. Arch Dermatol 1979;115:140915 .

6 Gilliam JN, Sontheimer RD. Distinctive cutaneous subsets in the spectrum of lupus erythematosus. F Am Acad Dermatol $1981 ; 4: 471-5$

\section{Painful knee: a dilemma}

Q1: What are the findings on the bone scan and the MRI scans?

The isotope bone scan after injection of the technetium-99 methylene diphosphonate shows significantly increased uptake of the radiopharmaceutical by the right femoral head and the proximal femur (fig 2). Coronal T1 and fat saturated T2, MRI images of both hips were obtained. On T1 weighted image there is reduced signal intensity noted in relation to the head, neck, and proximal right femur (fig 3A). On T2 weighted image, there is patchy increased signal intensity in the same areas. A small joint effusion is also noticeable on the right side on $\mathrm{T} 2$ weighted image (fig $3 \mathrm{~B}$ ). The acetabulum appears normal in both films.

\section{Q2: What is the diagnosis?}

Physical examination, MRI images, and the pattern of radionuclide uptake are highly suggestive of transient osteoporosis of the right hip. In majority of the cases suffering from this disorder, osteopenic changes are evident on plain film in the proximal femur few weeks from onset. In rare cases, no loss of bone density may be detected throughout the duration of the disease. This variant of transient osteoporosis has been called "transient bone marrow oedema syndrome"1 The differential diagnosis includes avascular necrosis, rheumatoid arthritis, osteomyelitis, septic arthritis, and reflex sympathetic dystrophy. However in view of the clinical details and laboratory investigations these can easily be out ruled. Our patient was treated with NSAIDs and non-weight bearing on the affected hip. She was able to partially weight bear after nine months and her condition fully resolved at 12 months without surgical intervention.

\section{Discussion}

Transient osteoporosis was initially described as a clinical syndrome, which affected women in the third trimester of pregnancy. It is characterised by pain and focal loss of radiodensity in the affected hip. Contralateral hip may be involved in $30 \%$ of the cases. This is a rare, self limiting disorder where function and bone density of the hip are restored in six to 12 months. Absence of any underlying medical condition or drug therapy separates it from 
"secondary osteoporosis". ${ }^{2}$ Since the earlier reports, it is now known that this condition may affect both males and females irrespective of their race, age, or sex. However only few cases have been documented with patients in their sixth decade of life, with an atypical presentation of referred pain from the hip joint. A symptom often leading to diagnostic confusion. If the examining clinician does not perform a careful and complete physical examination.

The symptoms of transient osteoporosis are of sudden onset of hip pain, inability to weight bear, and restriction of motion. These symptoms are generally associated with proximal femoral osteoporosis on plain radiographs and increased radionuclide uptake on isotope bone scan. Such features are the early manifestations of a number of possible differential diagnoses including avascular necrosis, septic arthritis, rheumatoid arthritis, tuberculosis, pigmented and villonodular synovitis, and neoplasm. However, the diagnosis is not difficult if the clinician is aware of the characteristic features.

There is controversy about the aetiology, pathophysiology, and the outcome of the disease. It has been shown that increased bone metabolism contributes to the accumulation of free water in the bone marrow, leading to raised intramedullary pressure. This in turn may cause venous stasis rather than obstruct arterial blood supply and lead to the marrow changes. Metabolic, viral, and neurological factors have been suggested as possible links to the aetiology. ${ }^{1}$ Transient osteoporosis has also been regarded as a possible variant of reflex sympathetic dystrophy and the early phase of avascular necrosis of the femoral head.

The three main diagnostic tools used in evaluating transient osteoporosis are conventional radiographs, technetium-99m radionuclide scans, and MRI scans. Plain radiographs are the least sensitive of the group. Typical changes of osteopenia with patchy demineralisation in the femoral head do not develop for four to six weeks, until then the radiographs remain normal. However these changes may not occur at all and it has been suggested that this may be a separate radiological entity or a variant of transient osteoporosis called "bone marrow oedema syndrome". ${ }^{3}$ This phenomenon was observed in our patient where no osteopenia was detected on standard film throughout the course of the disease, leading to diagnostic confusion for clinicians unaware of this entity. Radionuclide scan shows increased uptake in the femoral head and the proximal femur within days of onset, but it lacks the anatomical detail and is non-specific. However MRI scan is able to demonstrate the changes within the marrow, cortex, and the surrounding soft tissue with surprisingly good clarity. Three phases of transient osteoporosis have been identified on MRI scan. ${ }^{4}$ The initial "diffuse stage" with patchy hypointensities on the T1 weighted images with hyperintensive marrow oedema and joint effusion on $\mathrm{T} 2$ weighted images. This may be seen as early as 48 hours. Focal stage takes uptil three months to develop with hypointensive areas localised to the weight bearing regions of the femoral head. Finally the "residual phase", which occurs six to 12 months from the onset of symptoms displaying return to normal marrow intensity associated with resolution of symptoms.

Transient osteoporosis only affects epiphyseal and metaphyseal areas without affecting soft tissues. It has been suggested that transient osteoporosis is an early stage of AVN as initial MRI findings are similar in both cases and some documented cases of transient osteoporosis progressed to osteonecrosis. ${ }^{5}$ However in osteonecrosis the presence of double line sign on T2 weighted images representing an outer rim of lower intensity (zone of fibrosis) and an inner rim of hyperaemia (zone of granulation tissue) of high signal intensity is considered pathognomic. In addition on technetium-99m bone scan an area of avascular necrosis appears as a "cold defect" excluding transient osteoporosis. In comparison patients with septic arthritis, joint effusion, marrow oedema, and bony erosions appear as low signal intensity on $\mathrm{T} 1$ images within 24 hours of infection. However the final diagnosis requires culture of the aspirate. ${ }^{6}$ As the disease is self limiting the treatment remains NSAIDs, protected weight bearing, and an active and passive range of motion exercises. Core decompression of the femoral neck has been used in a small number of patients with reduction in recovery time. This procedure requires further evaluation to be recommended. ${ }^{5}$

A review of literature suggests that transient osteoporosis is not as rare as initially presumed and may often result in unnecessary expensive, prolonged and often painful diagnostic procedures for a self limiting condition. We feel it is important to include this condition in the differential diagnosis of hip or knee pain. The availability of MRI has not only improved the chances of early detection of this disorder but can also help in out ruling other more serious conditions.

\section{Final diagnosis}

Unilateral transient bone marrow oedema syndrome.

1 Ostlere SJ, Gold RH. Osteoporosis and bone density measurement methods. Clin Orthop 1991;271:149-58.

Wilson AJ, Murphy WA, Hardy DC, et al. Transient Wilson AJ, Murphy WA, Hardy DC, et al. Transient
osteoporosis: transient bone marrow oedema. Radiology osteoporosis: transien

3 Hayes CW, Conway WF, Daniel WW. MR imaging of the bone marrow edema pattern: transient osteoporosis, transient bone marrow edema syndrome, or osteoporosis. Radiographics 1993;13:1001-11.

4 Grimm J, Higer HP, Benning R, et al. MRI of transient osteoporosis of the hip. Arch Orthop Trauma Surg 1991;110:98102.

5 Turner DA, Templeton AC, Selzer PM, et al. Femoral capital osteonecrosis: MR findings of diffuse marrow abnormalities without focal lesions. Radiology 1989;171:135-40

6 Lay JP, Whitehouse RW. Painful hip in a patient on long Lay JP, Whitehouse RW. Painful hip in a patient on
term steroid treatment. Postgrad Med f 1998;74:693-4.

Back pain and dyspnoea in a middle aged diabetic male

Q1: What is the unifying diagnosis?

Pulmonary valve endocarditis (with septic pulmonary emboli and evidence of early right ventricular failure) secondary to $S$ aureus spinal osteomyelitis. 
The spinal osteomyelitis is the likely substrate for the endocarditis rather than vice versa as symptoms of back pain preceded cardiopulmonary symptoms by several weeks.

It is unclear whether the initial presentation elsewhere was with septic pulmonary emboli secondary to pulmonary valve endocarditis mimicking pneumonia, which was then partially treated, or whether he acquired $S$ aureus bacteraemia secondary to an intravascular catheter with subsequent development of osteomyelitis and endocarditis: a murmur was noted at the initial presentation but one set of blood cultures was negative.

The chest radiograph shows bilateral basal infiltrates due to septic pulmonary emboli. Magnetic resonance imaging of the thoracic spine showed evidence of disc infection at the D9/D10 level, with abnormal enhancement extending into the paravertebral spaces anteriorly and laterally. A transthoracic echocardiogram shows a large vegetation attached to the pulmonary valve by a stalk. In addition, pulmonary artery pressure was raised at $45 \mathrm{~mm}$ $\mathrm{Hg}$, there was moderate tricuspid regurgitation and paradoxical septal motion.

\section{Q2: How does right sided endocarditis (RSE) usually present?}

Presentation is usually with fever and symptoms caused by septic pulmonary emboli, which occur in $60 \%-100 \%$ of patients. ${ }^{1}$ These include pleuritic chest pain (present in $60 \%$ of intravenous drug users (IVDUs) with $\mathrm{RSE}^{2}$ ), cough, dyspnoea, and haemoptysis. The chest radiograph is abnormal in three quarters of patients at presentation. ${ }^{3}$ Abnormalities include infiltrates, often multiple and appearing sequentially with a predilection for the lower lobes, cavitation, pleural effusion, and empyema. ${ }^{4}$ Tricuspid and pulmonary valve dysfunction is usually well tolerated haemodynamically so that pleuropulmonary rather than cardiac features usually dominate the presentation.

While these features are likely to prompt consideration of the diagnosis in IVDUs, the diagnosis may not be considered in nonaddicted patients. In one series of 29 nonIVDUs eventually proved to have RSE, an average of nine months elapsed between onset of symptoms and diagnosis. Multiple admissions were common, positive blood cultures tended to be either disregarded as contaminants or ascribed to pulmonary events, and misdiagnoses such as pneumonia, malignancy, and vasculitis were frequent. ${ }^{5}$

Q3: What risk factors are recognised for right sided endocarditis in non-IVDUs?

The majority of these patients have chronic underlying medical conditions. ${ }^{5}$ Predisposing factors include congenital heart disease (whereas the majority of IVDUs with RSE have a structurally normal heart), inadequately treated sepsis, and indwelling vascular catheters (central lines, pacemakers, pulmonary artery catheters, etc).
Q4: What is the likelihood of endocarditis in cases of $S$ aureus bacteraemia?

Eighty per cent of cases of $S$ aureus bacteraemia are community acquired. With no other obvious primary source of bacteraemia, one third of these patients have underlying endocarditis. ${ }^{6}$ Nosocomially acquired cases are on the increase and usually occur in the context of intravenous cannulae/indwelling vascular devices. ${ }^{7}$ Factors known to increase the chance of underlying endocarditis in patients with $S$ aureus bacteraemia include absence of an alternative primary site of infection, community acquisition, and metastatic sequelae. $^{8}$ The incidence of endocarditis resulting in intravenous catheter related $S$ aureus bacteraemia is thought to be $<5 \%-10 \%{ }^{6}$ However, many cases may be overlooked: a recent study examining the utility of early transoesophageal echocardiography in patients with $S$ aureus bacteraemia (the majority of whom acquired it nosocomially) has demonstrated that vegetations are frequently identified in cases where endocarditis is not suspected clinically. ${ }^{9}$ These cases often affect the right side of the heart at sites of endocardial injury induced by the catheters, commonly in the absence of pre-existing valvular disease. ${ }^{7}$ In one series of 142 consecutively necropsied patients, 55 of whom had undergone pulmonary artery catheterisation during their final hospitalisation, right sided endocardial lesions (subendocardial haemorrhages and thrombi) were identified in over half of the 55 cases, with evidence of endocarditis in $7 \%$. Endocardial lesions were found in only $3 \%$ of the non-catheterised patients. $^{10}$

Q5: What is the relationship between back pain and endocarditis?

Musculoskeletal symptoms are a common but under-appreciated feature of endocarditis: in one series of 192 cases, $44 \%$ of patients had musculoskeletal manifestations (including back pain, arthralgias, arthritis, and myalgias), and were the sole presenting feature in $15 \%$. In the majority, musculoskeletal symptoms were present for some time before diagnosis and responded rapidly to treatment of the endocarditis. The incidence of back pain in this series was $12.5 \%$, with disc space infection identified in just under a quarter of these. ${ }^{11}$ In another series of 108 patients with endocarditis, 30\% had musculoskeletal manifestations with back pain an important feature in 13 of the 108 (in whom four cases of vertebral osteomyelitis were identified). In this study, patients presenting with musculoskeletal symptoms were diagnosed significantly later and had a significantly higher mortality, presumably because of delays in initiation of treatment. $^{12}$

Severe back pain may therefore be the predominant presenting complaint in a significant minority of cases of endocarditis and factors such as localised myositis, emboli, and immunological mechanisms have been cited to explain its presence in the absence 

Box 1: Factors predisposing to RSE
- Intravenous drug abuse
- Congenital heart disease
- Indwelling intravenous device
- Immunosuppression

\section{Box 2: Learning points}

- Right sided endocarditis presents with pleuropulmonary rather than cardiac symptoms.

- Incidence is increasing in non-addicted patients.

- Misdiagnoses such as pneumonia or malignancy are common.

- One third of patients with community acquired Staphylococcus aureus have underlying endocarditis.

- Metastatic infection occurs commonly in Staphylococcus aureus bacteraemia.

- Back pain may be a presenting feature of endocarditis but does not always indicate osteomyelitis or disc space infection.

of evidence of osteomyelitis or disc space infection. ${ }^{11} 12$

\section{Discussion}

RSE accounts for approximately $5 \%-10 \%$ of all cases of endocarditis and is predominantly a disease of IVDUs. ${ }^{4}$ The incidence is increasing both in this group and in non-addicted patients (the latter because of increasing use of intravascular catheters). $S$ aureus accounts for the majority of cases in IVDUs ${ }^{13}$; in the series of non-IVDUs cited earlier $S$ aureus, Streptococcus bovis, and candida were the commonest organisms identified. ${ }^{5}$

Pulmonary valve endocarditis is rare. In one combined series of 351 cases of endocarditis (both left and right sided) in IVDUs, isolated pulmonary valve endocarditis was found in only $1 \%$ of cases. A further $1 \%$ had combined involvement of the pulmonary and tricuspid valves. ${ }^{14}$ In another series of 132 IVDUs with RSE, involvement of the pulmonary or pulmonary and tricuspid valves occurred in five cases. ${ }^{13}$ As with tricuspid endocarditis, typical mucocutaneous features are unusual and a murmur may not be audible at presentation (In one series, a murmur was absent at the time of hospitalisation in $28 \%$ of a series of IVDUs with RSE. ${ }^{13}$ ) Diagnosis may therefore be difficult and the true incidence is probably underestimated. ${ }^{4}{ }^{15}$ Although transoesophageal echocardiography is superior to transthoracic echocardiography in the detection of valvular vegetations in left sided endocarditis, improved diagnostic accuracy has not been shown in tricuspid valve endocarditis. ${ }^{16}$ The situation is less clear for pulmonary valve endocarditis, though transoesophageal echocardiography may offer advantages over transthoracic echocardio- graphy. ${ }^{15}{ }^{17}$ As with tricuspid valve endocarditis, prognosis is favourable with a high response rate to medical treatment. ${ }^{4}$

$S$ aureus is the second commonest cause of native valve endocarditis, accounting for $30 \%$ of total cases and has an increasing incidence (though it accounted for fewer than 10\% of cases in the preantibiotic $\mathrm{era}^{18}$ ). It is the commonest cause of nosocomially acquired endocarditis and the commonest causative organism in IVDUs. ${ }^{2}$ It is notable in that it commonly presents as an acute rather than a subacute illness and frequently affects normal valves. (In a series of 106 cases, half of the nonIVDUs had no underlying cardiac disease; only one case of pulmonary valve involvement was identified. ${ }^{19}$ ) In addition, suppurative complications (such as myocardial abscess formation) are common with metastatic localisations occurring in $40 \%$ of cases. ${ }^{2}$ In a series from Denmark (where necropsy rates are high) over half of cases of $S$ aureus endocarditis were not suspected clinically and only diagnosed at postmortem examination. ${ }^{20}$

This case illustrates the fact that the correct diagnosis of RSE is rarely initially considered because of a presentation that is dominated by pleuropulmonary symptoms, an oversight particularly likely in non-IVDUs in whom a high index of suspicion is required. The case also illustrates the propensity for $S$ aureus to metastasise and reinforces the importance of immediately considering the possibility of endocarditis in any patient with $S$ aureus bacteraemia, particularly if community acquired, even in the absence of signs of cardiac disease. Clinicians should remain mindful that musculoskeletal symptoms are common in endocarditis, may dominate the clinical picture in a significant minority of patients, and may lead the unwary away from the true diagnosis.

The patient was treated with a prolonged course of intravenous antibiotics. He remains well at follow up with improvement in his exertional dyspnoea and gradual resolution of his back pain.

\section{Final diagnosis}

Pulmonary valve endocarditis secondary to $S$ aureus spinal osteomyelitis.

Factors predisposing to RSE and learning points are shown in boxes 1 and 2 .

We are grateful to Sharon Lynn, senior cardiac technician at Queen Mary's Hospital, for providing a photograph of the echocardiographic appearance of the pulmonary valve.

1 Remetz MS, Quagliarello V. Endovascular infections arising from right sided heart structures. Cardiol Clin 1992;10:137-49. 2 Saccente M, Cobbs CG. Clinical approach to infective endocarditis. Cardiol Clin 1996;14:351-62.

3 Chambers HF, Miller RT, Newman MD. Right sided Staphylococcus aureus endocarditis in intravenous drug abusers: two week combination therapy. Ann Intern Med 1988;109:619.

4 Panidis IP, Kotler MN, Mintz GS. Right heart endocarditis: clinical and echocardiographic features. Am Heart $\mathcal{f}$ 1984;107:759-64.

5 Nandakumar R, Raju G. Isolated tricuspid valve endocarditis in non addicted patients: a diagnostic challenge. Am $\mathcal{F}$ Med Sci 1997;314:207-12.

6 Mortara LA, Bayer AS. Staphylococcus aureus bacteraemia and endocarditis. Infect Dis Clin North Am 1993;7:53-68.

7 Fernandez-Guerrero ML, Verdejo C, Azofra J, et al. Hospital acquired infectious endocarditis not associated with cardiac surgery: an emerging problem. Clin Infect Dis 1995;20: 16-23. 
8 Bayer AS, Lam K, Ginzton L, et al. Staphylococcus aureus bacteraemia. Clinical, serological and echocradiographic findings in patients with and without endocarditis. Arch Intern Med 1987;147:457-62.

9 Fowler VG Jr, Li J, Corey GR, et al. Role of echocardiography in evaluation of patients with Staphylococcus aureus bacteraemia: experience in 103 patients. $\mathscr{f} \mathrm{Am}$ Coll Cardio 1997;30:1072-8

10 Rowley KM, Clubb KS, Walker Smith GJ, et al. Right sided infective endocarditis as a consequence of flow directed pulmonary valve catheterisation. N Engl f Med 1984;311:1152-6.

11 Churchill MA, Geraci JE, Hunder GG. Musculoskeletal manifestations of bacterial endocarditis. Ann Intern Med 1977;87:754-9.

12 Thomas P, Allal J, Bontoux D, et al. Rheumatological manifestations of infective endocarditis. Ann Rheum Dis 1984;43: $716-20$

13 Hecht SR, Berger M. Right sided endocarditis in intravenous drug users. Ann Intern Med 1992;117:560-6.

14 Reisberg BE. Infective endocarditis in the narcotic addict. Prog Cardiovasc Dis 1979;22:193-204.

15 Shapiro SM, Young E, Ginzton LE, et al. Pulmonic valve endocarditis as an underdiagnosed disease: role of transoesophageal echocardiography. F Am Soc Echocardiogr 1992; 5:48-51.

16 San Roman JA, Vilacosta I, Zamorano JL, et al. Transoesophageal echocardiography in right sided endocarditis. f Am Coll Cardiol 1993:21:1226-30.

7 Am Coll Cardiol 1993;21:1226-30.
7 Winslow T, Foster E, Adams JR, et al. Pulmonary valve endocarditis: improved diagnosis with biplane transoesophageal echocardiography. If $\mathrm{Am}$ Soc Echocardiogr 1992;5:206-10.

18 Sanabria TJ, Alpert JS, Goldberg R, et al. Increasing frequency of staphylococcal infective endocarditis. Arch Intern Med 1990;150:1305-9.

19 Watanakunakorn C. Staphylococcus aureus endocarditis at a community teaching hospital, 1980-1991. An analysis of 106 cases. Arch Intern Med 1994;154:2330-5.

20 Espersen F, Frimodt B, Moller N. Staphylococcus aureus endocarditis: a review of 119 cases. Arch Intern Med 1986;146:1118-21.

\section{A man with abdominal pain}

Q1: What is the unusual feature on the plain abdominal $x$-ray?

The plain abdominal $x$-ray shows the presence of hepatic-portal venous gas (HPVG) (fig 2). The distribution of gas is towards the periphery of the liver, as opposed to gas in the biliary tree which is central in distribution. Distended loops of small bowel are also present.

Q2: What are the causes of this feature? The conditions in which HPVG has been reported are listed in box $1 .^{12}$

\section{Q3: Is laparotomy indicated}

The patient underwent laparotomy, which revealed ischaemic bowel in the distribution of the superior mesenteric artery. Attempts to revascularise the bowel failed and the patient died in the early postoperative period.

HPVG is not a specific disease entity and is merely another diagnostic clue in patients with acute abdominal pathology. HPVG is associated with various diverse conditions and its importance must be derived from the clinical context of the patient.

Treatment is therefore directed at the underlying cause. The majority of patients will have ischaemic bowel (72\%) and will require exploratory laparotomy. However, patients with ulcerative colitis without other indication for laparotomy will not require exploration. ${ }^{3}$

\section{Discussion}

HPVG is an uncommon $x$-ray finding. It was first described in infants in $1955^{1}$ and in adults in $1960 .{ }^{4}$ It appears as a branching radiolucency extending to within $2 \mathrm{~cm}$ of the liver capsule and $x$-rays are most revealing when

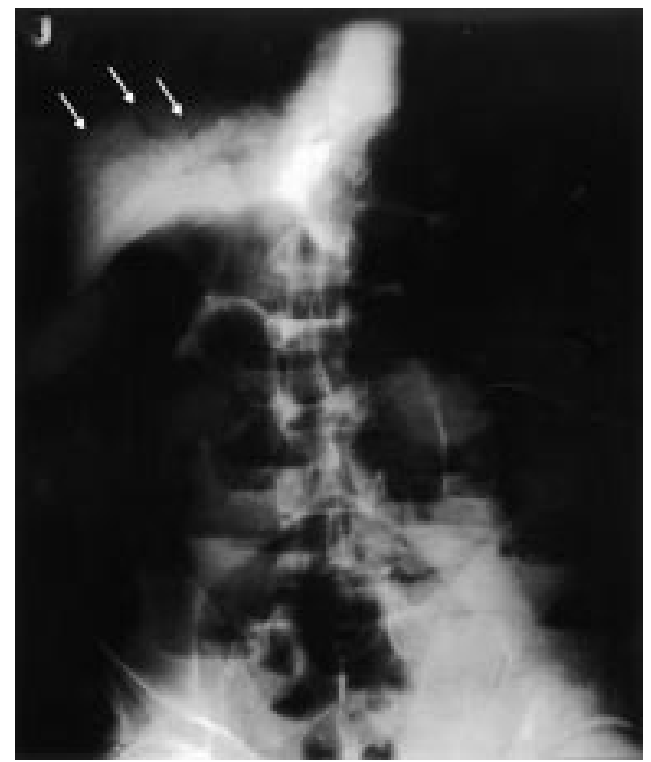

Figure 2 Abdominal x-ray.

Box 1: Reported causes of HPVG

Non-iatrogenic

- Necrotic bowel (72\%)

- Ulcerative colitis/Crohn's disease $(8 \%)$

- Intra-abdominal abscess (6\%)

- Bowel obstruction (3\%)

- Peptic ulcer

- Acute haemorrhagic pancreatitis

- Diabetic ketoacidosis

- Necrotising enterocolitis

- Unknown

Iatrogenic

- Recent contrast enema

- Endoscopy/ERCP

- Recent liver transplantation

taken in the left lateral decubitus position. The difference in distribution of gas in the biliary tree and portal venous gas is due to the fact that gas in the biliary tract is carried towards the porta hepatis by the centripetal flow of the bile and therefore appears central in the liver while the HPVG travels peripherally enhanced by the centrifugal flow of the blood. ${ }^{4}$

The factors that are thought to predispose to HPVG are ${ }^{5}$ : mucosal damage, as seen in intestinal infarction, inflammatory bowel disease, and peptic ulcer disease; bowel distension as in small bowel obstruction; and sepsis due to bowel necrosis, spreading cellulitis or intraabdominal abscess, although these factors are not identified in every patient.

With the increased use of computed tomography and ultrasound, HPVG has also been reported from iatrogenic causes, most of which will not require laparotomy, ${ }^{2}$ eg, recent contrast enema, endoscopy, ERCP, and post-liver transplantation.

\section{Final diagnosis}

Hepatic-portal vein gas due to ischaemic bowel. 


\section{Box 2: Learning points}

- HPVG on plain abdominal $x$-ray is uncommon.

- The $x$-ray pattern of HPVG must be differentiated from that of biliary tract gas.

- HPVG can be found in a variety of clinical settings.

- The need for laparotomy is determined from the clinical context of the patient.

1 Wolfe JN, Evans WA. Gas in the portal veins of infants: a roentgenographic demonstration with post-mortem anatomical correlation. $A \mathcal{F R} 1955 ; 74: 486-9$

2 Hong JJ, Gadaleta D, Rossi P, Esquivel J, Davis JM. Portal vein gas, a changing clinical entity. Arch Surg 1997;132: $1071-5$

3 Traverso LW. Is hepatic portal venous gas an indication for exploratory laparotomy? Arch Surg 1981;116:936-8.

4 Susman N, Senturia HR. Gas embolisation of portal venous system. AfR 1960;83:847-50.

5 Liebman PR, Patten MT, Manny R, Benfield JR, Hechtman

5 Liebman PR, Patten MT, Manny R, Benfield JR, Hechtman physiology and clinical significance. Ann Surg 1978;187: 281-7.

\section{A shot in the dark}

Q1: What abnormalities are demonstrated on the plain radiographs of the wrist?

Radiograph of the left wrist (fig 1) shows an air gun pellet in the soft tissues of the forearm but, in addition, there is widening of the distal radial growth plate and irregularity, lucency and cupping of the radial and ulnar metaphyses consistent with rickets. The carpal bone age is delayed, at approximately 4 years, but a radiograph of the left hand showed a dissociation between the carpal bone age and that of the remainder of the hand. This can occur due to intercurrent illness and, in isolation, is not indicative of globally delayed skeletal maturation. The differential diagnosis of the wrist radiograph includes rickets secondary to vitamin $\mathrm{D}$ deficiency, an abnormality of vitamin D metabolism, target cell abnormalities, including renal tubular disorders, or tumour-induced rickets, and metaphyseal chondrodysplasias (box 1).

\section{Box 1: Causes of metaphyseal fraying,} cupping, and lucency

Rickets secondary to:

- Nutritional factors

- Target cell abnormalities including renal tubular disorders

- Tumour-induced rickets

- Hepatic disease

Hereditary abnormalities of vitamin D metabolism:

- Metaphyseal chondrodysplasias

- Copper deficiency

- Wilson's disease
Q2: What biochemical tests would aid the diagnosis?

Biochemical investigation is aimed at assessing the degree of renal impairment and characterising the nature of the renal abnormality. Serum analysis revealed mild renal impairment with a mild acidosis and consequent mild secondary hyperparathyroidism: urea $6.5 \mathrm{mmol} / 1$ (normal range 2.5-6.5 mmol/l), creatinine 112 $\mathrm{mmol} / \mathrm{l}$ (30-80), bicarbonate $18 \mathrm{mmol} / \mathrm{l}(20-$ 26), sodium $134 \mathrm{mmol} / 1$ (132-145), potassium $3.4 \mathrm{mmol} / 1$ (3.5-5.5), glucose $8.9 \mathrm{mmol} / 1$ (2.58.0 ). The serum parathyroid hormone was mildly raised with normal serum calcium and marginally low serum phosphate at 0.97 $\mathrm{mmol} / \mathrm{l}(1.00-1.80)$. There was no serological evidence of copper deficiency or Wilson's disease which can give these radiological appearances and the latter can also result in a Fanconi syndrome.

Urine analysis revealed a severe tubular aminoaciduria with evidence of mild tubular acidosis (total serum bicarbonate $18 \mu \mathrm{mol} / 1$ with a urine $\mathrm{pH}$ of 7) but the biochemical and clinical features did not correspond to a particular proximal or distal tubular defect. The biochemical and radiological features did not correspond to renal tubular acidosis (RTA) type I, in which there is severe hypokalaemia, nephrocalcinosis and, most often, an acute presentation, nor with RTA type IV in which there is hyperkalaemia. ${ }^{12}$ The absence of Fanconi syndrome of glycosuria, aminoaciduria and hyperphosphaturia also excluded RTA type II. Examination of the eyes did not demonstrate tapetoretinal degeneration which, with the lack of a salt-losing nephropathy, excluded the autosomal recessive Senior Loken syndrome which is a major cause of end-stage renal failure in children, accounting for $10-20 \%$ of cases. The late presentation and clinical features excluded vitamin D-dependent and X-linked vitamin D-resistant rickets, Lignac-Fanconi syndrome (cystinosis), and oxalosis.

\section{Q3: What additional radiological} investigation should be performed and what abnormalities might be expected? In rickets secondary to renal causes, ultrasound and dimercaptylsuccinic acid (DMSA) scan may show features suggestive of a cause of renal failure, for example, small echogenic kidneys due to renal parenchymal disease, scarring, or reflux nephropathy. Nephrocalcinosis may be seen as part of RTA

\section{Box 2: Learning points}

- The commonest causes of rickets are dietary, followed by renal disease

- Always look objectively at the whole radiograph

- If there is a metaphyseal abnormality, follow a diagnostic pathway to differentiate the causes 


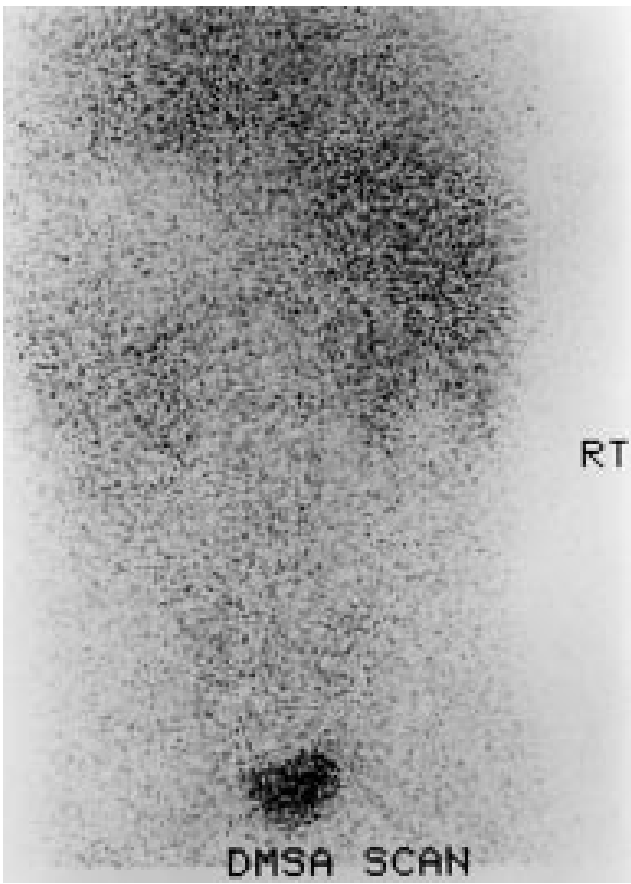

Figure 2 DMSA scan of the kidneys.

type I. In our patient, ultrasound showed that the kidneys were small for age (below the 5 th centile), and mildly echogenic, with poor cortico/medullary differentiation but no definite evidence of nephrocalcinosis. The finding of a renal abnormality in conjunction with the results of serum and urine biochemistry excluded the diagnosis of rickets due to hepatic or nutritional disorders of vitamin D metabolism and also excluded primary metaphyseal chondrodysplasias.

A DMSA scan was performed to see if the cause of the small echogenic kidneys was reflux nephropathy. The scan showed almost no uptake of DMSA by the kidneys (fig 2). This can be due to inability to bind DMSA within the kidney or occasionally due to poorly functioning kidneys where the serum creatinine is over $300 \mathrm{mmol} / \mathrm{l}$, ie, much higher than in this case. DMSA binds to plasma protein and, intracellularly, to cytosol proteins and mitochondria. Uptake occurs principally in the cells of the proximal tubules. Nonvisualisation of the kidneys with DMSA is, therefore, an indication of the functioning state of the tubules. It was therefore concluded that these findings were indicative of mild chronic renal failure due to renal dysplasia in which there was a tubular defect leading to severe aminoaciduria and therefore, poor DMSA binding. It was not possible to exclude scarring on a DMSA scan with such poor DMSA uptake.

\section{Discussion}

In chronic renal disease, metaphyseal abnormality results from failure of the kidneys to hydroxylate 25-hydroxy-vitamin $\mathrm{D}_{3}$ and diminishing capacity to excrete phosphate. This stimulates production of parathyroid hor- mone, osteoclastic activity and bone resorption, resulting in temporary correction of serum calcium and phosphate. ${ }^{3}$ The serum calcium and phosphorus may be maintained within the normal range until the glomerular filtration rate goes below $25-30 \mathrm{ml} / \mathrm{min}$ when persistent hyperphosphataemia occurs. The growth plates, whilst appearing widened on plain radiographs, are actually narrowed when examined histologically with a region of osteitis fibrosa generalisata adjacent to the growth plate. This gives the widened growth plate appearance that simulates rickets. The growth plates are weak and susceptible to fracture resulting in a slipped epiphysis, although this is not usually seen until 11-16 years of age. Unlike renal osteodystrophy in adults, it is unusual in children to see phalangeal subperiosteal erosion and soft tissue and arterial calcification.

Management is aimed at reducing the degree of hyperparathyroidism, improving calorie intake and long-term monitoring of renal impairment. This case illustrates the importance of keeping an open mind when reporting trauma radiographs and a diagnostic pathway for evaluating metaphyseal abnormality.

\section{Final diagnosis}

Renal rickets secondary to non-specific renal protein-losing tubular abnormality.

1 Thomas PS. Metabolic bone disorders. In: Carty H, Shaw D, Brunelle F, Kendall B, eds. Imaging children. New York: Churchill Livingstone, 1994:992-1011.

2 Taybi H. Radiology of syndromes and metabolic disorders. Chicago: Year Book Medical Publishers, 1983; pp 338-339.

3 Sundaram M. Renal osteodystrophy. Skeletal Radiol 1989; 18:363-7.

\section{Pyrexia and pancytopenia with unusual} host immune response

\section{Q1: What cytomorphological}

phenomenon is shown in fig 1 ?

Figure 1 shows a proliferation of benign histiocytes in the bone marrow smears. They have abundant foamy to granular cytoplasm and many of them contain phagocytosed blood cells and their precursors. This cytomorphological phenomenon is called haemophagocytic histiocytosis and can be seen in the bone marrow, lymph nodes, spleen and liver in a variety of clinical settings.

\section{Q2: List the conditions with which this cytomorphological phenomenon may be associated}

The conditions associated with haemophagocytic histiocytosis are listed in box 1 .

\section{Q3: What is the diagnosis in this case?}

Figure 2 shows greater detail of the histiocyte. Numerous $2-4 \mu \mathrm{m}$ round to oval bodies with eccentric to central nuclei and a perinuclear vacuole are identified within the cytoplasm. These were morphologically identified, on silver methenamine and periodic acid Schiff 


\section{Box 1: Clinical states associated with} haemophagocytosis

Primary

- Familial haemophagocytic lymphohistiocytosis

- Virus-associated haemophagocytosis (with no prior immunodeficiency)

Secondary/reactive

- Malignancies, especially T cell lymphomas

- Acquired and hereditary immunodeficiency

- Parenteral fat overload

- Panniculitis

- Bacterial, parasitic and fungal infections (tuberculosis, typhoid, brucellosis, malaria, leishmaniasis, histoplasma, etc) stain, as yeast forms of histoplasma. Figure 2(A) also shows phagocytosed red cell precursors.

\section{Discussion}

Haemophagocytic syndrome is a clinicopathological state characterised by fever, blood cytopenias, coagulopathy, hepatosplenomegaly and a systemic proliferation of mature histiocytes showing phagocytosis of blood cells. ${ }^{1}$ The diagnostic guidelines as laid down by the Histiocytic Society ${ }^{1}$ are shown in box 2 . Haemophagocytosis (HP) is a disorder of the cellular arm of immune response occurring as a consequence of unrestricted monocyte activation that results from marked elevation of inflammatory cytokines produced by dysregulated reactive or neoplastic $\mathrm{T}$ lymphocytes. ${ }^{2}$ Profound cytopenias, which may result from haemophagocytosis or associated coagulopathy, may seriously compromise the already morbid patient.

First described as a familial disease by Farquhar and Claireaux in $1952{ }^{3}$ haemophagocytic syndrome has subsequently been reported to occur in a host of other clinical settings (box 2$)^{5-9}$; it is also called secondary or reactive haemophagocytosis. ${ }^{5}{ }^{6}$ Haematological malignancies, especially $\mathrm{T}$ cell lymphomas, have been reported as the most frequent cause of reactive HP in most studies. ${ }^{7}$ Infections are an important association in tropical countries, in the oriental population ${ }^{6}$ and, with increasing incidence of AIDS, in the West as well. ${ }^{8}{ }^{9}$

Histoplasmosis has been sporadically reported from different parts of India. The commonest manifestation in the Indian population is involvement of skin and mucous membranes, with or without systemic involvement. ${ }^{10}$ The organism has been identified in various sites, including skin, mucous membranes, blood, bone marrow, lymph nodes, and sputum. ${ }^{10}$ However, HP in a setting of disseminated histoplasmosis has, to the best of our knowledge, not been described previously from India. The few reported cases in English literature have occurred mostly in
Box 2: Diagnostic guidelines for haemophagocytic lymphohistiocytosis ${ }^{1}$

Clinical and laboratory criteria

- Fever ( $\geqslant 7$ days; peaks $\geqslant 38.5^{\circ} \mathrm{C}$ )

- Splenomegaly ( $\geqslant 3 \mathrm{~cm}$ below costal margin)

- Cytopenia (affecting $\geqslant 2$ of three lineages in the peripheral blood not caused by hypocellular or dysplastic bone marrow)

- Haemoglobin $<9 \mathrm{~g} / \mathrm{dl}$

- Platelets $<100 \times 10^{9} / 1$

- Neutrophils < $1.0 \times 10^{9} / 1$

- Hypertriglyceridaemia and/or hypofibrinogenaemia ${ }^{\star}$

Morphological criteria

- Haemophagocytosis in bone marrow or lymph nodes or spleen

* Some of these guidelines may not apply to a secondary haemophagocytic state ${ }^{2}$

patients with AIDS. ${ }^{89}$ In the wake of an alarming increase in the number of AIDS cases in the sub-continent, the importance of careful laboratory examination in the diagnosis of histoplasmosis cannot be overemphasised, particularly because, given the high prevalence of tuberculosis in India, cases of pulmonary or disseminated histoplasmosis may be misdiagnosed as tuberculosis. Presence of severe cytopenias in a patient with prolonged pyrexia should alert the physician to the possibility of HP and a meticulous search in the bone marrow and other reticuloendothelial sites should be made for the underlying cause of HP. An early diagnosis of infection-associated reactive HP is crucial in the management of this potentially reversible condition.

\section{Final diagnosis}

Disseminated histoplasmosis with reactive haemophagocytosis.

1 Henter JI, Elinder G, Ost A, et al. Diagnostic guidelines for the hemophagocytic lymphohistiocytosis. Semin Oncol 1991; 18:29-33.

2 Favara BE. Hemophagocytic lymphohistiocytosis: a hemophagocytic syndrome. Semin Diagn Pathol 1992;9:63-74.

3 Farquhar JW, Claireaux AX. Familial haemophagocytic Farquhar JW, Claireaux AX. Familial ha
reticulosis. Arch Dis Child 1952;27:519-25.

reticulosis. Arch Dis Child 1952;27:519-25.
Risdall RJ, McKenna RW, Nesbit ME, et al. Virus associated hemophagocytic syndrome. Cancer 1979:44:993-1002.

5 Arya S, Hong R, Gilbert EF. Reactive hemophagoctic syndrome. Pediatr Pathol 1985;3:129-41.

6 Wong KF, Chan JKC. Reactive hemophagocytic syndrome. A clinicopathological study of 40 patients in an oriental population. Am f Med 1992;93:177-80.

7 Linn YC, Tien SL, Lim LC, et al. Hemophagocytosis in bone marrow aspirate. A review of the clinical course of 10 cases. Acta Haematol 1955;94:182-91.

8 Koduri PR, Chundi V, De Marais P, Mizock BA, Patel AR, Weinstein RA. Reactive hemophagocytic syndrome: a new presentation of disseminated histoplasmosis in patients with AIDS. Clin Infect Dis 1995;21:1463-5.

9 Kurtin PJ, McKinsey DS, Gupta MR, Dirks M. Histoplasmosis in patients with acquired immunodeficiency syndrome-hematologic and bone marrow manifestations. Am f Clin Pathol 1990;93:367-72.

10 Mukherjee AK, Mukherjee D, Mukopadhyay M. Histoplasmosis in India. A clinicopathological review with report of a case in a child. Indian f Pathol Microbiol 1986;29:26370 . 


\section{Abdominal colic after vigorous exercise in a middle aged man}

Q1: What is the diagnosis?

The diagnosis is coeliac band with coeliac artery stenosis causing chronic intestinal ischaemia. This is such a rare clinical condition that even institutions with an enormous experience in peripheral vascular surgery have accumulated some experience over several years. It is only by reflex consideration of this condition after excluding other common diagnosis that the alert surgeon will reach this diagnosis.

Q2: What are the differential diagnoses in the patient?

The main differential diagnoses include mesenteric angina that may be secondary to atherosclerosis, Buerger's disease, fibromuscular dysplasia, and arteritis such as Takayasu's disease. Other possibilities include renal and biliary colic.

\section{Q3: How would you establish the diagnosis?}

This patient's presentation was atypical of common abdominal conditions and the routine investigations were normal. With a history such as this, atherosclerotic disease of the coeliac/mesenteric arteries causing chronic mesenteric ischaemia was a possibility. Measuring the erythrocyte sedimentation rate would help to rule out an inflammatory cause. A duplex scan would be performed as the first step of vascular imaging as this is noninvasive. ${ }^{1}$ However because of their anatomical location, assessment of visceral arterial lesions by duplex scan is not as reliable as it is in other situations. Contrast computed tomography (fig 1) or magnetic resonance angiography of the coeliac/mesenteric arteries, provide better information including detection of any coexisting extrinsic pathology. Finally the definitive investigation is an angiogram, which in this case clearly demonstrated pathology at the origin of the coeliac artery. This was left until last due to its invasive nature and the risks, although rare, associated with it.

Q4: What do the investigations illustrate? The angiogram demonstrates a tight stenosis (lateral film) (fig 2) at the origin of the coeliac artery. This may be due to extrinsic or intrinsic factors or a combination of both; the superior mesenteric artery (SMA) is of normal calibre. The inferior mesenteric artery (IMA) is not seen.

\section{Q5: What are the treatment options available?}

Treatment options should be based on risk versus benefit for each procedure. In this patient median arcuate ligament (coeliac band) syndrome had to be ruled out as an extrinsic cause of compression. The risk of releasing the band is much less than arterial reconstruction. At operation he was found to have a coeliac band. This was divided, however a thrill over the coeliac artery persisted. This indicated the presence of dual pathology, with stenosis of the coeliac artery being also present. In this case the stenosis was localised to the proximal portion of the coeliac artery; arterial reconstruction between the aorta and hepatic artery was seen as the best treatment option. The SMA was of normal calibre. The IMA was found to be small and could possibly have contributed to the patient's symptoms as the collateral circulation had failed to develop. A reversed long saphenous vein graft was anastomosed between the infra renal aorta and the hepatic artery and the graft was tunnelled behind the pancreas.

Aortocoeliac grafting is an option, however due to the short course of the coeliac artery, the procedure is often difficult. Another option is to reimplant the coeliac artery into the aorta at another site; this is particularly suitable if there is a tight proximal stenosis with an aortic component. ${ }^{2}$ Angioplasty with or without stenting of the mesenteric vessels is a further option, the risk being distal embolisation and dissection with acute thrombosis causing ischaemia or infarction.

When reviewed in the clinic at three months and six months the patient was completely free from symptoms and has returned back to his normal exercise programme. Contrast computed tomography performed at three months confirmed the patency of the aortohepatic graft.

\section{Discussion}

It has been reported in the literature that up to $33 \%$ of the population have an anatomical variation in the structure of the coeliac axis. ${ }^{3}$ Such anomaly seldom gives rise to symptoms of chronic intestinal ischaemia. The coeliac axis, SMA, and IMA along with the iliacs provide the main blood supply to the gastrointestinal tract. Due to the presence of a good collateral circulation, stenosis usually has to be of a severe degree before symptoms occur. This can be graded by measuring the pressure gradient across the stenotic lesion. Stenoses likely to compromise the collateral circulation may become clinically manifested during the postprandial phase or during muscular exertion. It has been also demonstrated that mesenteric vascular resistance increases during exercise and produces a marked decrease in arterial inflow. ${ }^{4}$

Chronic gastrointestinal ischaemia is classically described as presenting with postprandial abdominal pain (termed visceral angina), weight loss, and an epigastric bruit (quoted in over $80 \%$ of cases). Lord et al, in their series of 12 patients with coeliac axis compression, noted that $75 \%$ (9) of their patients had atypical abdominal pain, 58\% (7) of the patients had slight weight loss, 
and only $42 \%$ (5) had history of diarrhoea. ${ }^{5}$ The presentation in this patient was unusual in that he had no postprandial symptoms, but pain was induced by heavy exercise perhaps due to redistribution of blood flow. In the presence of coeliac artery stenosis and a small IMA the collateral blood flow to the gut through the internal iliac arteries was possibly diverted to the lower limbs during exercise. Since the SMA was patent, the patient had very little symptoms of weight loss, diarrhoea, or postprandial symptoms.

\section{Final diagnosis}

Coeliac band and coeliac artery stenosis.

1 Perko MJ. Importance of diastolic velocities in detection of celiac and mesenteric artery disease by duplex ultrasound. $\mathcal{F}$ Vasc Surg 1997;26:288-93.

2 Takach TJ. Celiac compression syndrome: tailored therapy based on intraoperative findings. F Am Coll Surg 1996;183: 606-10.

3 Lindner HH, Kemprud E. A clinicoanatomical study of the arcuate ligament of the diaphragm. Arch Surg 1971;103: $600-5$

4 White S, Patrick T, Higgins CB. Effects of altering ventricular rate on blood flow distribution in conscious dogs. Am $\mathcal{F}$ Physiol 1971;221:1402.

5 Lord RSA, Sydney MD, Stoney RJ, et al. Coeliac-axis compression. Lancet 1968;ii:795-8.

\section{International Postgraduate Diary}

23rd European Conference on Psychosomatic Research

17-21 June 2000: Oslo, Norway

Details: Congress-Conference AS - CONGREX, Thomas Heftyes gt. 2, PO Box 2694 Solli, N-0204 Oslo, Norway. Tel +47 (0) 2256 1930; fax + 47 (0) 2256 0541; email: ecpr2000@congrex.no

Falk Symposia

4-6 May 2000: Hepatology 2000 (Munich, Germany)

9/10 June 2000: Cholestasis and gallstones (Cluj Napoca, Romania)

1/2 October 2000: Non-neoplastic diseases of the anorectum - an interdisciplinary approach (Freiburg, Germany)

3/4 October 2000: Immunosuppression in inflammatory bowel diseases-standards, news, and future trends (Freiburg, Germany)
12/13 October 2000: Biology of bile acids in health and disease (Den Haag, The Netherlands)

4 November 2000: Chronic inflammatory bowel diseases-progress and controversies at the turn of the century (Bucharest, Romania)

Details: Falk Foundation eV-Congress Division, Leinenweberstr 5, PO Box 6529, D-79041 Freiburg, Germany. Tel +49 (0) 761 130340; fax +49 (0) 761 1303459; email: symposia@falkfoundation.de

Columbia University College of Physicians and Surgeons, New York

5/6 May 2000: 12th Annual orthopaedic trauma course. Current techniques in upper \& lower extremity trauma

22-25 May 2000: 4th Annual conference. Botanical medicine in modern clinical practice
28-31 July 2000: 10th Annual corse. A comprehensive review of movement disorders for the clinical practitioner

30 July-5 August 2000: 5th Annual course. Update and intensive review in internal medicine

Details: Center for Continuing Education, Columbia University College of Physicians and Surgeons, 630 West 168th Street, Unit 39, New York, NY10032, USA. Tel + 1212 781 5990; fax + 1212781 6047; email: cme@columbia.edu

Ninth International Symposium on celiac disease

10-13 August 2000: Hunt Valley, MD, USA

Details: Althea Pusateri, Program Coordinator, University of Maryland School of Medicine, $655 \mathrm{~W}$ Baltimore Street, Baltomore, MD 21201, USA Tel +1 410706 3957; fax +1 410 706 3103; http://www.celiaccenter.org 